\title{
Structure of the pre-outburst accretion disk in SS Cygni
}

\author{
D. A. Kononov ${ }^{1}$, F. Giovannelli ${ }^{2}$, I. Bruni ${ }^{3}$, and D. V. Bisikalo ${ }^{1}$ \\ 1 Institute of Astronomy of the Russian Academy of Sciences, 48, Pyatnitskaya str., 119017 Moscow, Russia \\ 2 INAF - Istituto di Astrofisica Spaziale e Fisica Cosmica - Roma, Area di Ricerca di Tor Vergata, via del Fosso del Cavaliere 100, \\ 00133 Roma, Italy \\ e-mail: franco.giovannelli@iasf-roma.inaf.it \\ 3 INAF Osservatorio Astronomico di Bologna, via Ranzani 1, 40127 Bologna, Italy
}

Received 15 December 2010 / Accepted 10 November 2011

\section{ABSTRACT}

\begin{abstract}
We present results of spectroscopic observations of SS Cygni performed at the $1.5 \mathrm{~m}$ telescope of the Loiano Observatory in June 2009, three days before a long outburst. Using results of these observations, we computed Doppler tomograms in three Balmer lines $\mathrm{H}_{\beta}, \mathrm{H}_{\gamma}$, and $\mathrm{H}_{\delta}$. Analyzing the tomograms, we identified gas dynamic features existing in the system. It is shown that in the preoutburst disk a number of shock waves exist. These waves are "hot line" a wave produced by the interaction between the circum-disk halo and the stream from the inner Lagrangian point $\mathrm{L}_{1}$, and two arms of the spiral tidal shock and bow shock caused by the motion of the accretor and disk in the gas of the circum-binary envelope. We also found that before the outburst the density and size of the disk increase.
\end{abstract}

Key words. techniques: spectroscopic - accretion, accretion disks - stars: dwarf novae - stars: individual: SS Cygni novae, cataclysmic variables

\section{Introduction}

The SS Cygni system belongs to the dwarf nova class of cataclysmic variables (CVs). Dwarf novae are characterized by two states of activity, quiescence, and outburst. There are several models proposed for describing the physical mechanisms leading to outbursts. The most popular of them are the secondary instability model (SIM, Bath 1973) and disk instability model (DIM, Abramowicz et al. 1995). Another mechanism was proposed in Bisikalo et al. (2004), in which the increase in the accretion rate is caused by the formation of a spiral density wave in the inner regions of the dense disk. These models have their advantages in particular cases but still cannot explain all of the reasons for outbursts in cataclysmic variables.

To reveal the physical conditions in the disk during both of these states, many observational studies have been performed. Reviews of SS Cygni discussing its multi-frequency behavior and models include those of Giovannelli \& Martinez-Pais (1991), Giovannelli (1996), and Giovannelli \& Sabau-Graziati (1998). Among the spectroscopic studies of SS Cygni, we highlight the two studies of Martinez-Pais et al. (1994) and MartinezPais et al. (1996). The second is focused on a detailed analysis of outburst spectra of SS Cygni and their evolution with time. In this work, it was shown that some spectra and their behavior are consistent with the SIM model in terms of the observed features of outbursts, which some DIM models and other observational data do not display. Thus, the question of which physical mechanisms lead to outbursts remains open. Since it is known that the outburst is triggered by a higher accretion rate, to answer this question, it is necessary to investigate the flow structure in the system.

One of the methods for investigating the flow structure is Doppler tomography (Marsh \& Horne 1988). This method transforms the orbital variability of emission-line intensities into luminosity maps in the two-dimensional velocity space $\left(V_{x}, V_{y}\right)$. In the papers of Bisikalo et al. (2008); Kononov et al. (2008); and Boneva et al. (2009), SS Cygni was investigated using Doppler tomography in addition to three-dimensional (3D) gas dynamic simulations. This approach allowed the authors to identify the main gas dynamical elements existing in the system in the quiescent state (immediately after an outburst) and at a late stage of the outburst.

In this paper, we propose new spectroscopic data of SS Cygni acquired three days before a long outburst. Using them, we aim to investigate the structure of the pre-outburst disk in SS Cygni. In addition, we use spectroscopic data, tomograms, and the results of the gas dynamic simulations of Bisikalo et al. (2008). They were acquired at the beginning of the quiescent state immediately after a short outburst. Comparing these results with the new ones, we attempt to derive conclusions about the changes occurring in the disk during quiescence.

\section{Observations and data reduction}

The observations were performed on 18 June 2009 using the Bologna Faint Object Spectrometer and Camera (BFOSC) (Merighi et al. 1994) mounted at the Cassegrain focus of the $1.52 \mathrm{~m}$ "Cassini" Telescope of the Bologna Astronomical Observatory. The BFOSC is equipped with a $1300 \times 1340$ pixels EEV D129915 CCD detector (pixel size $20 \times 20 \mu \mathrm{m}^{2}$, FOV $13 \times 13 \mathrm{arcmin}^{2}$, pixel scale $\left.0.58 \mathrm{arcsec} / \mathrm{pixel}\right)$. The main dispersion element of the spectrograph is a grism with the resolving power of $3.9 \AA\left(\sim 270 \mathrm{~km} \mathrm{~s}^{-1}\right.$ at $\left.4340 \AA\right)$, corresponding to $\Delta \lambda /$ pixel of $1.7 \AA /$ pixel. The spectral range covered is 3300 $5350 \AA$ A.

Spectral processing was performed with the "MIDAS long" package. The processing routine included the following steps. 

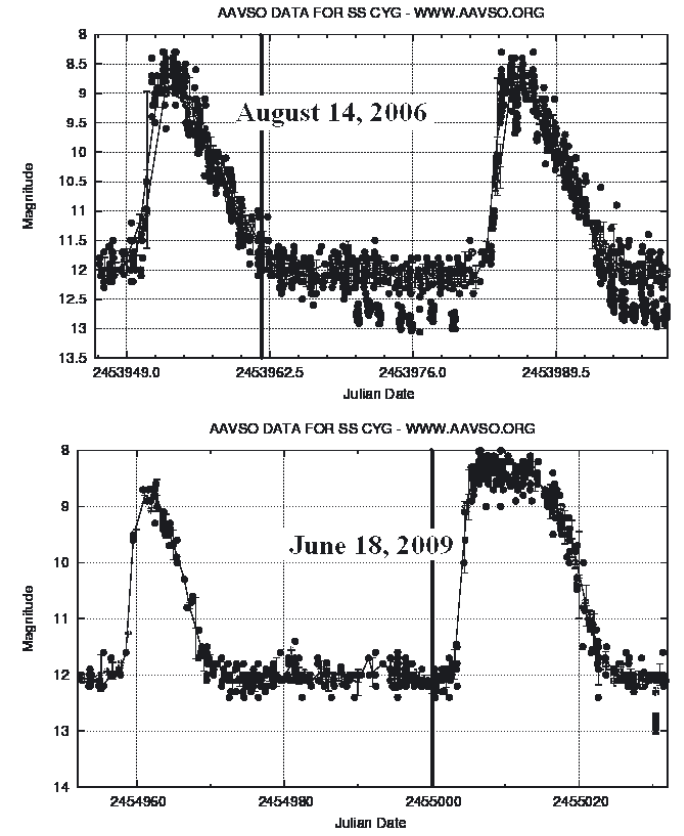

Fig. 1. Light curves of the SS Cygni system taken from the AAVSO web page with the observational nights marked.

Photometric data reductions consisted of the BIAS and flat-field calibrations and sky background subtraction. The flat-field calibration was performed in the pixel-to-pixel mode. To perform the wavelength calibration, we prepared arc-lamp frames as follows. The initial spectra were obtained in four-five subsets per night. The arc-lamp was exposed before and after each subset. Studying the arc-lamp frames, we found that the same spectral (He-Ar) lines were shifted slightly from frame to frame, thus we prepared a particular WL-calibration frame for each subset. To perform this we averaged the arc-lamp frames in sets of two from the start to the end of the subset. Using the resulting arc-lamp frames, we determined the dispersion relations and calibrated one-dimensional (1D) object spectra. After the wavelength calibration, we chose several spectral lines of interest. As a result, we obtained series of spectral line profiles - in sets of 53 of the $\mathrm{H}_{\beta}, \mathrm{H}_{\gamma}$, and $\mathrm{H}_{\delta}$ lines for the night of June 18, 2009. We referenced the spectra to the phase of the binary using the ephemeris of North et al. (2002)

$T_{0}=2450622.5483(2)+0.27512973\left( \pm 12^{-8}\right) E(\mathrm{HJD})$,

where $T_{0}$ is the epoch when the radial velocity passes through zero from negative to positive values, and following North et al. (2002), we took the orbital period from Hessman et al. (1984), to be $E$ the cycle number since the zero point. For observations made in 2009, $E=15915$, and 53 profiles of each line cover a binary phase interval from 0.75 to 0.25 . The mean inverse dispersion of the spectra is $\sim 1.7 \AA$ /pixel.

The observational data of August, 2006, mentioned in Sect. 1, were obtained using the 2-m telescope of the observatory located at Terskol peak (Caucasus, Russia). Observations and data reduction were described in Bisikalo et al. (2008). To preserve the uniformity of the data in both the sets, we used the same reduction procedure for both of them.

In Fig. 1, we show light curves of the system taken from the AAVSO web page ${ }^{1}$ with the observational nights marked.

\footnotetext{
${ }^{1}$ http://www . aavso.org/
}

Looking at this figure, one can see that the short outburst of August 2006 was followed by another short outburst, while the outburst of June 2009 we discuss here was long. This causes some additional questions about the validity of the comparison we discuss in detail in Sect. 4.

\section{Doppler tomograms}

Using our acquired trailed spectra, we calculated Doppler tomograms of SS Cygni. To perform Doppler tomography, we used our own implementation of the maximum entropy algorithm proposed by Lucy (1994). The entropy term that we used was identical to that proposed in the quoted paper. The default maps were floating, and were obtained by smearing the iterated image with a Gaussian function. We took the same width of the smearing function for all the tomograms to ensure that they could be accurately compared. The initial and restored trailed spectrograms and corresponding tomograms are shown in Figs. 2 and 3 for $\mathrm{H}_{\beta}$ and $\mathrm{H}_{\gamma}$, and $\mathrm{H}_{\delta}$, respectively.

The root-mean-square (rms) error in the tomogram restoration was $\sim 7 \%$ for all the tomograms. To calculate the rms, we restored the profiles from the obtained tomograms to the same phases as those of the initial spectra, calculated the rms error of each individual spectrum, and then for each tomogram calculated a simple average of the individual errors. In Fig. 4, one can compare the average initial and restored spectra. Both of the lines in each panel are normalized to their total intensity.

The total intensity of each map is normalized to unity. The dashed line denotes the Roche lobes of the donor and accretor. The line with the circles is part of the stream trajectory. The line with the filled diamonds traces disk velocities at points located along the stream. In each tomogram, a ring-like structure is clearly visible. It means that the disk exists in the system. All the tomograms display an asymmetry. To make it clearer we divided each map into quadrants. In the $\mathrm{H}_{\beta}$ and $\mathrm{H}_{\gamma}$ tomograms, there are spots in the upper- and lower-left quadrants $\left(V_{x}<0\right)$. In $\mathrm{H}_{\delta}$, the bright region in the lower-left quadrant $\left(V_{x}, V_{y}<0\right)$ resembles a spot, but the bright region in the upper-left quadrant extends to both the right quadrants and finishes in the asymmetric region.

In standard disks of CVs, the temperature increases inwards. Thus, spectral lines with higher excitation energies should originate in regions closer to the accretor. For the considered quasiKeplerian disks, the velocity also increases inwards, hence lines with higher excitation energies should occupy tomograms regions with higher velocities. By examining Figs. 2 and 3, we can clearly see that for lines with higher excitation energies the tomograms have larger sizes. In addition, by studying Figs. 2, 3 (panels with trailed spectrograms), and Fig. 4 one can see that the line width also grows for the lines with higher excitation energies, i.e. from $\mathrm{H}_{\beta}$ to $\mathrm{H}_{\delta}$.

In the considered set of the lines, the $\mathrm{H}_{\delta}$ line has the highest excitation energy, whereas the $\mathrm{H}_{\beta}$ has the lowest. Therefore, the $\mathrm{H}_{\delta}$ tomogram (Fig. 3) has the largest size; the $\mathrm{H}_{\gamma}$ tomogram (Fig. 2, right) has a medium size, and the $\mathrm{H}_{\beta}$ tomogram (Fig. 2, left) has the smallest.

However, central parts of the tomograms behave in a slightly different way. We see that the size of the central part of the $\mathrm{H}_{\gamma}$ tomogram is larger than that of $\mathrm{H}_{\beta}$. This proves that in the $\mathrm{H}_{\gamma}$ line we can see deeper layers of the disk that are optically thick to $\mathrm{H}_{\beta}$. The size of the central part of the $\mathrm{H}_{\delta}$ tomogram is comparable with the size of the central part of $\mathrm{H}_{\beta}$. This may be explained in the following way. If we assume that the bright spots in the tomograms are shock waves (e.g. Bisikalo et al. 2008), one can see that in the $\mathrm{H}_{\delta}$ tomogram the shock waves have the highest 

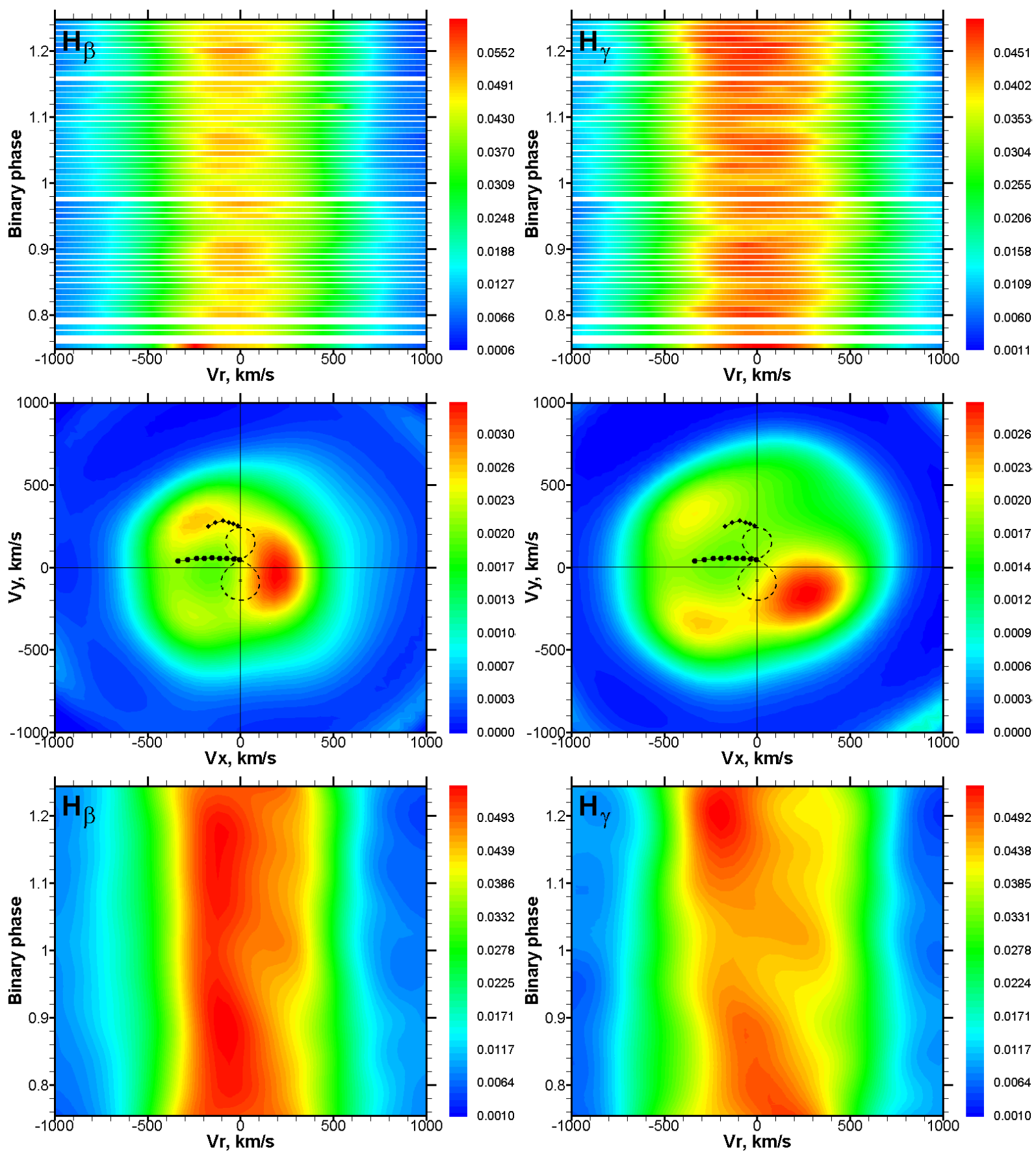

Fig. 2. Initial trailed spectra (set of June 18, 2009), Doppler tomograms, and corresponding restored trailed spectra of the lines $\mathrm{H}_{\beta}$, and $\mathrm{H}_{\gamma}$. Dashed line denotes the Roche lobe of the donor and accretor stars. The asterisk denotes the accretor. The line with the circles is part of the ballistic trajectory of a particle moving from the $\mathrm{L}_{1}$ point. The line with the diamonds traces disk velocities along the stream.

intensity relative to the background. Thus, probably, in $\mathrm{H}_{\delta}$ we see mostly the radiation of the shock waves. These waves are located in the outer regions of the disk (Bisikalo et al. 2004, 2008), where the Keplerian velocities of gas are small.

\section{Results and discussion}

To explain the observed details of the tomograms, we consider the results of gas dynamic simulations performed following the approach of Bisikalo et al. (2008). In Fig. 5, an intensity distribution over the equatorial plane in the spatial coordinates and the $\mathrm{H}_{\beta}$ Doppler map are shown.
The intensity distribution is calculated using the results of gas dynamical simulations described in Bisikalo et al. (2008). It corresponds to the quiescent state of the system. The markers in the upper panel denote the following features of the flow pattern: two lines with triangles correspond to the arms of the spiral tidal shock; the line with the hollow diamonds denotes the "hot line", a shock that occurs during the interaction of the circumdisk halo with the stream from the $\mathrm{L}_{1}$ point; and the line with the filled diamonds lies in the region along the stream from the $\mathrm{L}_{1}$ point. In the case of large disks and more intensive circumdisk halos, the "hot line" begins in regions edging the stream nearer to the $\mathrm{L}_{1}$ point (Bisikalo et al. 1998). The line with the filled diamonds schematically denotes the beginning of such a "hot line". 

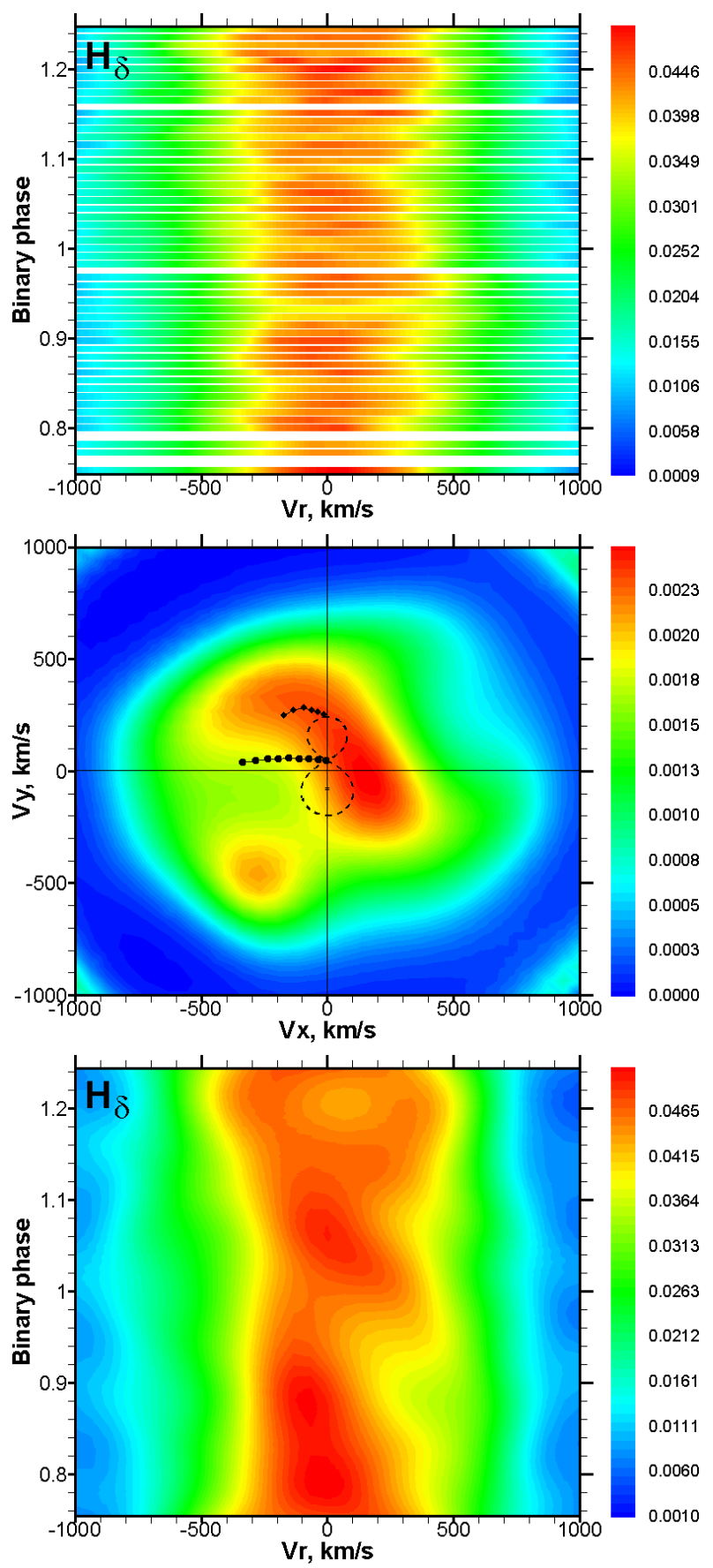

Fig. 3. Initial trailed spectra (set of June 18, 2009), Doppler tomograms, and corresponding restored trailed spectra of the line $\mathrm{H}_{\delta}$. The markers are the same as in Fig. 2.

Following the idea of Bisikalo et al. (2008), we transferred the markers into the Doppler map to verify gas dynamics that exist in the pre-outburst disk of SS Cygni. In the lower panel of Fig. 5, the $\mathrm{H}_{\beta}$ Doppler map is shown. We can see that there is some correspondence between the markers and locations of the bright regions in the tomogram. However, the bright spots have slightly smaller velocities than the corresponding markers. The "hot line" lies near the bright region in the upper-left quadrant of the tomogram. The bright region in the lower-left quadrant is produced by the radiation of one arm of the tidal shock. Another arm of the spiral tidal shock is invisible, although this may be because its radiation overlaps with the radiation of the
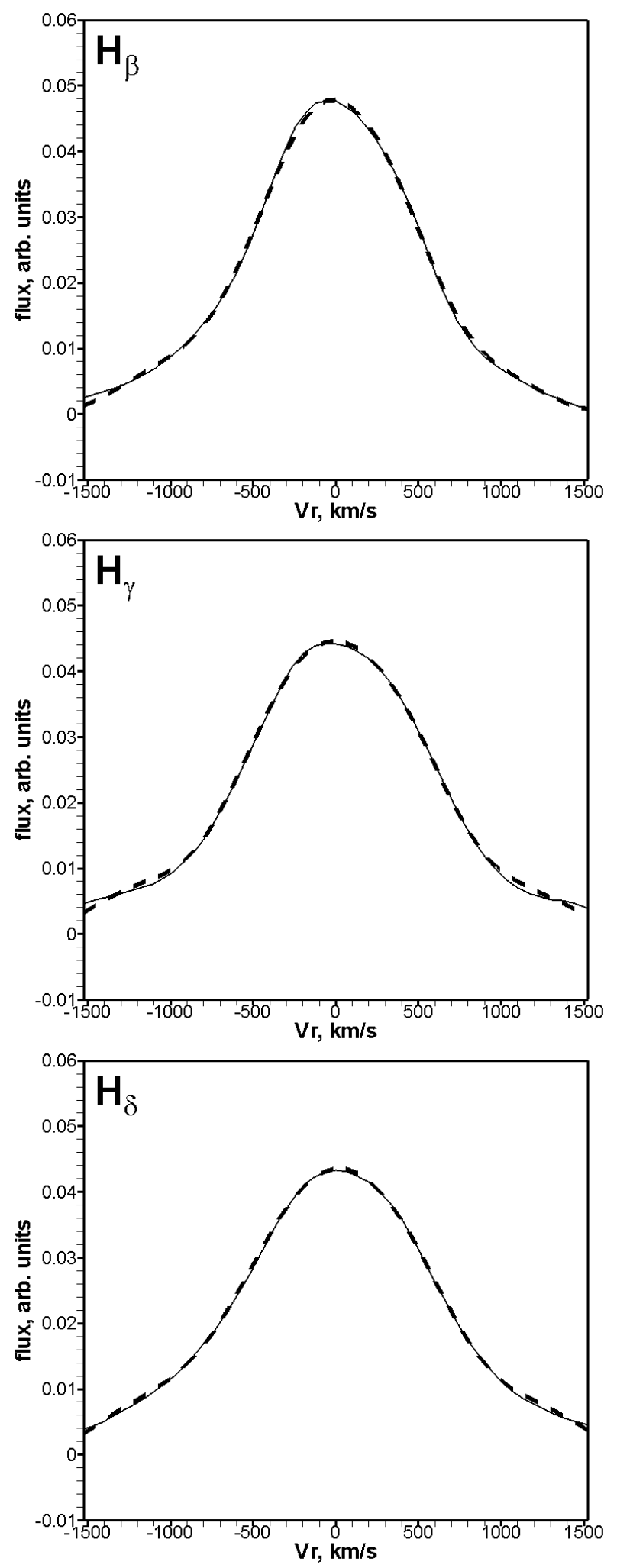

Fig. 4. The average initial (solid lines) and restored (dashed line) profiles of $\mathrm{H}_{\beta}, \mathrm{H}_{\gamma}$, and $\mathrm{H}_{\delta}$.

bow shock (see the next paragraph). The tidal spirals are observed in a number of dwarf novae and resolved using Doppler tomography (Papadimitriou et al. 2005; Papadaki et al. 2008). In gas dynamical simulations, they were firstly reported in Sawada et al. (1986). 

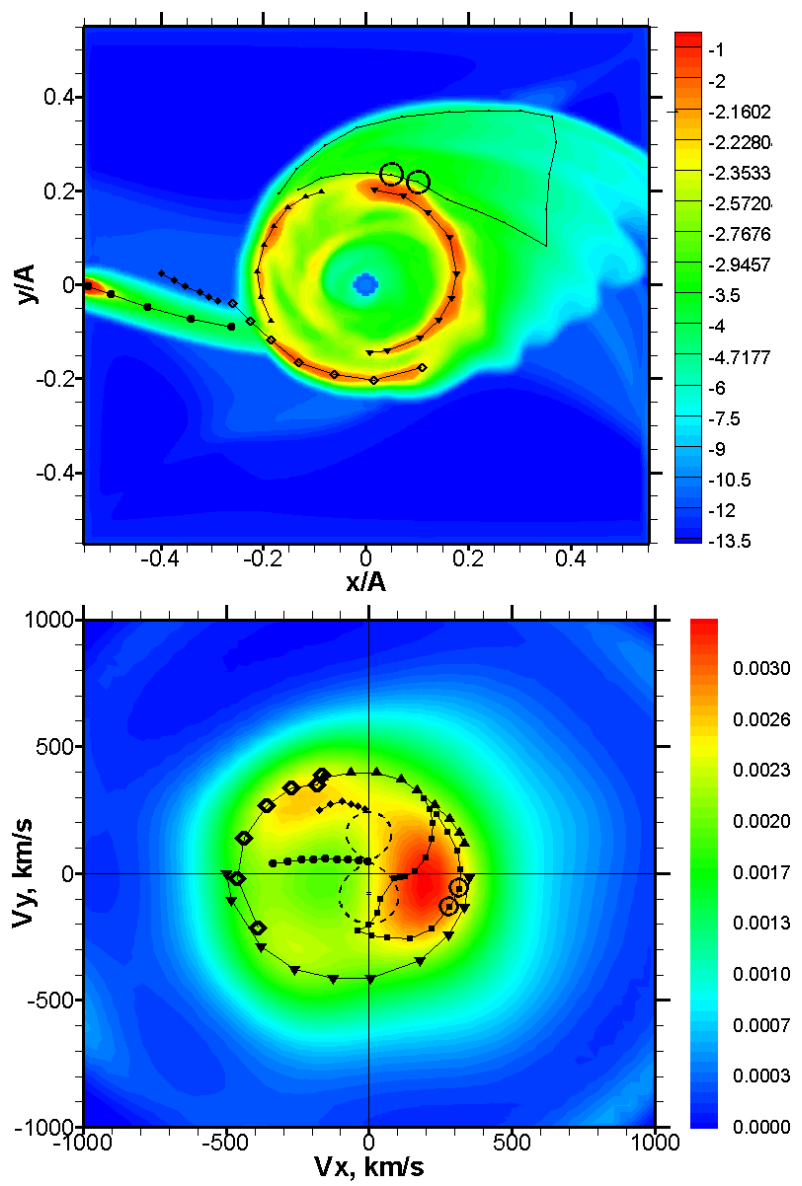

Fig. 5. Intensity distribution in the spatial coordinates (Bisikalo et al. 2008) (upper panel) and $\mathrm{H}_{\beta}$ Doppler map with the markers denoting main gas dynamic features. The markers in the upper panel denote the following gas dynamic features: lines with triangles correspond to the arms of the spiral tidal shock; the line with hollow diamonds denotes the "hot line"; the line with the filled diamonds lies in the region along the stream from the $\mathrm{L}_{1}$ point; and the thin solid line encloses the region of the matter near the bow shock. Two open circles on this line are placed to mark a boundary between the disk and asymmetric region (see explanation in the text). In the lower panel, we plot the same markers transferred into the velocity.

One of the brightest details in the observed tomograms is an asymmetric region at small velocities (upper- and lower-right quadrants). The same asymmetric region was reported in North et al. (2001), who emphasized that it resides in $\mathrm{H}_{\alpha}$ tomograms of EM Cygni and SS Cygni and assumed that it may be either due to some flows that are outside the orbital plane, or even the propeller effect. Several authors report some indirect signs of a magnetic field in SS Cyngi (Gaudenzi et al. 2011, and the references therein; Gnedin et al. 1995), hence the propeller effect could have been in the system. However, the propeller effect affects the matter that has significant proper velocities since it works in the inner regions of the accretion disk (Zhilkin \& Bisikalo 2011). Therefore, the radiation originating in this matter from the inner regions of the disk must contribute to the tomograms at high velocities. The asymmetry that we observe is located at low velocities (the very inner regions of the tomograms), hence, it is difficult to believe that the propeller effect may be a reason for the reported asymmetry of the tomograms. An explanation of the asymmetry in flows located somewhere out of the equatorial plane is also rather doubtful. Our gas dynamical simulations show that the disk is geometrically thin and there is no significant flows far from the equatorial plane, while in tomograms we see that the relative brightness of the asymmetric region is rather high. In the results of 3D gas dynamical simulations (Bisikalo et al. 2008), one can clearly see that there is only one region of the flow pattern where matter has appropriately small velocities and (relative to the background) high density to produce such an asymmetric feature in the tomograms. This is a region of the matter accumulated behind the bow shock. Hence, we believe that the most natural explanation of the observed asymmetry of the tomograms is the existence of this extended region and, hence, the bow shock.

To study the properties of the accretion disk, we analyzed the spectral lines and compared the obtained flow pattern to the data acquired immediately after an outburst in 2006.

Before deriving any conclusions, it is necessary to explain the assumption that this comparison is valid, since the compared observations are three years apart. In addition, in 2006, the observations had been obtained after a short outburst, followed by another short outburst, while in 2009 the system was observed before a long outburst.

It is evident that an ideal case would be to perform observations during quiescence between two subsequent outbursts. Unfortunately, we do not have such a homogeneous set of observational material. Therefore, from our previous data we selected observations obtained immediately after a short outburst that corresponds (or similar) to the short outburst preceding observations of 2009, based on parameters such as its duration and amplitude. Since the short outbursts of 2006 and 2009 are similar to all the observed parameters, one would expect the mass and radius of the disk to be approximately the same after both of them. Even if this were not so, differences between these parameters should not be significant, since the AAVSO data (Fig. 1) illustrate that the system returns to approximately the same states (same observed magnitudes) after both outbursts. It would then be natural to assume that after an outburst the disk starts to accumulate mass and changes its macroscopic parameters. If, an instability then develops at a certain moment, the next outburst starts. If this instability develops shortly after the end of the previous outburst, the next one will be short (since the total disk mass is low). If the time that passed is long, the next outburst will be long, since the disk accumulates enough mass before the long and powerful outburst. Following this reasoning, we assume that the short outburst that preceded our observations in 2009 was equivalent to that of 2006 when we had acquired observations at the beginning of quiescence and in our case were able to see how disk properties change between short and long outbursts.

To study the behavior of the spectral lines, we measured their equivalent widths $(E W)$. Our measurements show that the mean $E W$ of the $\mathrm{H}_{\gamma}$ line observed on June 18, 2009 exceeds the same parameter of the $\mathrm{H}_{\gamma}$ line observed on August 14, 2006 by a factor of $\approx 2.5$. The mean $E W$ of $\mathrm{H}_{\gamma}$ in the new observations is $\sim 37 \AA$ and in observations of August 2006 is $\sim 15 \AA$. For the $\mathrm{H}_{\beta}$ line, the ratio of the $E W \mathrm{~s}$ is smaller (a factor of $\sim 1.4$ ). The mean $\mathrm{H}_{\beta}-E W$ in the observations of June 2009 is $\sim 35 \AA$ and in observations of August 2006 it is $\sim 26 \AA$. Thus, we see that the $E W$ s of the lines observed on June 18, 2009 (before the outburst) are larger then those of August 14, 2006 (after the outburst).

This effect may be explained in two ways: i) since we normalize our data using the continuum level, the differences in this level may cause the differences in the $E W \mathrm{~s}$; ii) before the outburst, the disk density increases.

We assume that the emission lines that we observe are of the recombination type. In this case, their intensity is proportional 


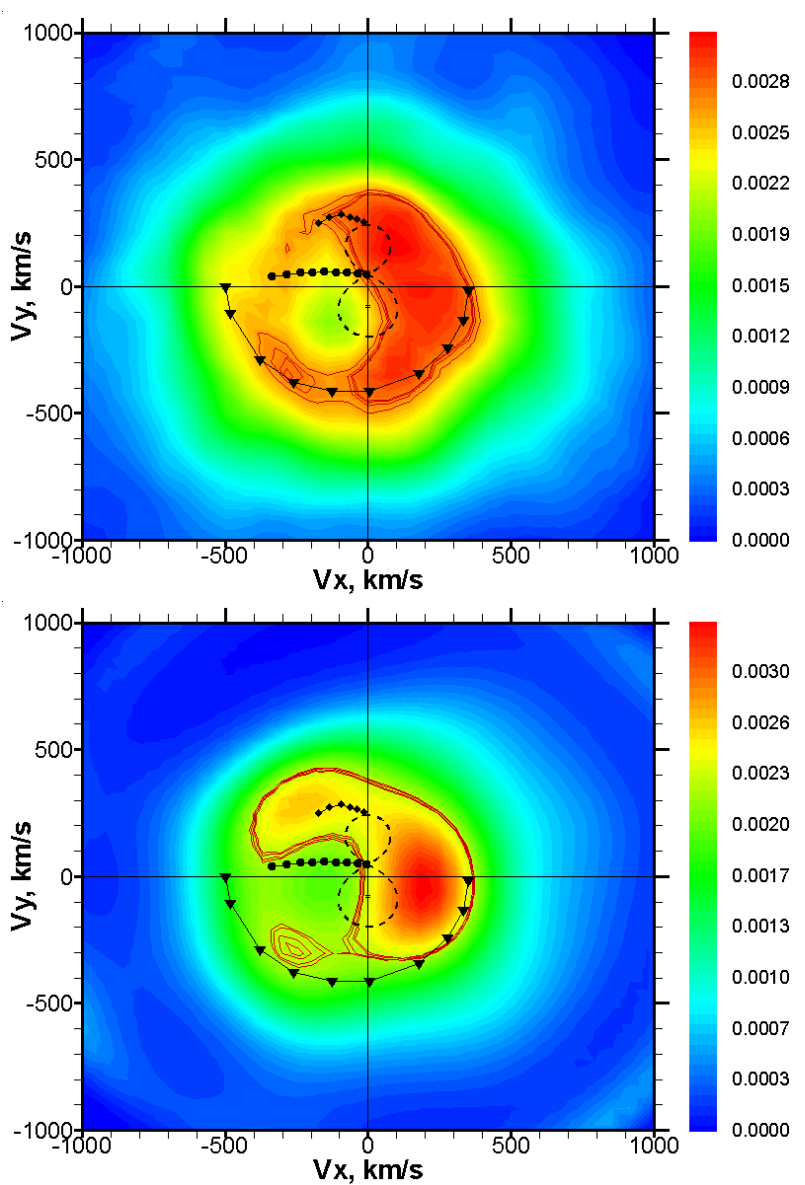

Fig. 6. Doppler tomograms in the $\mathrm{H}_{\beta}$ line. The upper panel is computed using the data of August 2006, while the lower panel is computed using the new data. Markers are the same as in Figs. 2 and 5. Certain isolines are plotted to select regions of tidal shock (lower left quadrants of the tomograms).

to $\rho^{2}$ (Storey \& Hummer 1995), where $\rho$ is the density. Hence for the same level of the continuum, the $E W$ increase may be explained by the higher density of the disk. Unfortunately, we performed neither absolute measurements of the flux nor simultaneous photometry in the B band to make quantitative conclusions. However, we can present some arguments supporting these hypotheses based on our analyses of the Doppler tomograms by plotting two tomograms in the $\mathrm{H}_{\beta}$ line of August 2006 (upper panel) and June 2009 (lower panel), as shown in Fig. 6. To ensure that any comparison of the tomograms is valid, we computed the tomogram of August 2006 using the same ME code with the same form as the default and the same width of smearing function.

The first remarkable difference between the two tomograms is that of size. In the tomogram of August 2006 we see that the disk down to the innermost regions has larger Keplerian velocities, while in the tomogram of 2009 these regions are invisible owing to the higher density and, hence, larger optical depth of the disk matter. On the basis of the spectral UV observations of SS Cygni, Sanad (2011) proposed the idea that the disk accumulates mass during quiescence. This is in agreement with the suggestion of Gaudenzi et al. (1990) that explains the behaviour of UV HeII lines in terms of the destruction of the disk during long outbursts and then its reformation during quiescence.

In Fig. 6, we have again plotted the markers, corresponding to one of the arms of the tidal shock wave, and marked the bright regions of the tomograms, which are believed to occur because of this shock, with isolines. There is one clear additional difference in the tomograms. We see that the region on the tidal shock of the tomogram of 2006 is located at slightly larger velocities. It must then be located closer to the accretor than the same region in the tomogram of 2009. The observed difference between the tomograms may mean that in June 2009 we observed a larger disk.

There is one clearly distinguishable feature in the new tomogram, which is denoted by the filled diamonds in Fig. 5. The filled diamonds lie in the region along the stream. If the disk increases in size as we have said above, the "hot line" occurs closer to the $\mathrm{L}_{1}$ point, because the larger disk and circumdisk halo collide with the stream close to this point. Thus, the presence of this detail is additional evidence of the larger disk.

The regions of the asymmetry marked in Fig. 5 by thin solid lines also differ between the tomograms. The outer boundary of the asymmetric region in the tomogram corresponds to its inner boundary in spatial coordinates, i.e. to the outer edge of the accretion disk. To illustrate this, we placed two points enclosed by circles in Fig. 5. As we can see in Fig. 6, in the tomogram of August 2006 (upper panel) the region of the asymmetry extends to $\sim 500 \mathrm{~km} \mathrm{~s}^{-1}$ along the $V_{x}$-axis, while in the tomogram of June 2009 its edge has the $V_{x}$-velocity lower than $400 \mathrm{~km} \mathrm{~s}^{-1}$. This means that the disk has different sizes at these two moments. On August 14, 2006, we observed the system immediately after the outburst when a significant portion of the disk matter had been accreted, and we can safely assume that the disk was smaller at that time. Three days before the outburst, on June 18, 2009, the disk after accumulating material during the quiescent period became larger, thus its outer edge and the region of the asymmetry had smaller velocities, as can be seen in the lower panel of Fig. 6 .

One additional difference between the asymmetric regions of the pre- and post-outburst tomograms is their shape and size. As we can see in Fig. 6 in the tomogram of the pre-outburst disk (lower panel), the asymmetric region is compact. Since this feature in the tomograms is produced by the radiation of the matter enclosed between the disk and bow shock, we can assume that in the steady-state pre-outburst disk the bow shock is located closer to the disk. Thus, the velocity gradients in the asymmetric region are shallower. In the post-outburst tomogram (upper panel), the asymmetric region is more extended. During the outburst, the accretion rate increases. At the same time, to remove the excess of the angular momentum, the outflow from the disk also increases (Sytov et al. 2007), finally leading to the growth of the region between the disk and bow shock. The matter in this region now has a wider range of velocities. Hence the region of the asymmetry in the post-outburst tomogram becomes more extended.

\section{Conclusions}

In June 2009, we performed spectral observations of SS Cygni, three days before an outburst. As a result, we obtained trailed spectra of the $\mathrm{H}_{\beta}, \mathrm{H}_{\gamma}$, and $\mathrm{H}_{\delta}$ lines, each containing 53 line profiles. This allowed us to generate the Doppler tomograms of SS Cygni in these lines and investigate the structure of the preoutburst disk.

The derived spectra and Doppler tomograms were compared with data acquired in August 2006 (Bisikalo et al. 2008) immediately after an outburst. This allowed us to draw some conclusions about changes in the disk parameters occurring during the quiescent state.

Analyzing the new Doppler tomograms and the results of gas dynamical simulations, we discern the main gas dynamical 
elements as shocks ("hot line", tidal spirals, bow shock) and that the region of matter behind the bow shock - found in previous works - remains in the disk and is located almost at the same positions. However, there are some significant differences between the new tomograms and those of August 2006. The sizes of the bright circular regions in these tomograms are smaller than in the tomograms of August 2006, meaning that in these new tomograms we cannot see the innermost regions of the disk because of its higher density and consequently larger optical depth. A comparison of the equivalent widths of the spectral lines also shows that the matter density in the disk increases with time.

The new tomograms show that the disk radius increases. One of the observations supporting this idea is the presence of a relatively bright region along the stream in the new tomograms. This means that the larger disk or circumdisk halo collides with the stream closer to the $\mathrm{L}_{1}$ point producing the "hot line" (an extended shock wave) closer to $\mathrm{L}_{1}$. In the tomogram, part of this "early hot line" is visible as the bright region with disk velocities corresponding to points located along the stream. One of the arms of the tidal shock in the new $\mathrm{H}_{\beta}$ tomogram is located at smaller velocities than in the tomogram of August 2006. This may provide support to the idea that the disk becomes larger before the outburst .

The outer edge of the asymmetric region in the pre-outburst tomogram of June 18, 2009 (Fig. 5, lower panel) is located at smaller velocities. This proves that the disk radius increases during the outburst.

Acknowledgements. This work was supported by the RAS, the Russian Foundation for Basic Research (project Nos. 09-02-00064, 09-02-00993), the Federal Targeted Program Science and Science Education for Innovation in Russia 20092013. We would like to thank the Bologna Astronomical Observatory for the allocation of telescope time, for excellent technical assistance, and for logistic support. We have appreciated the suggestions of the unknown referee that allowed us to improve the paper. Many thanks for this. Finally, many thanks to Mrs Claire Halliday, language editor, for cleaning our lame English. This research made use of NASA's Astrophysics Data System.

\section{References}

Abramowicz, M. A., Chen, X., Kato, S., Lasota, J.-P., \& Regev, O. 1995, ApJ, 438, L37

Bath, G. T. 1973, Nature Phys. Sci., 246, 84
Bisikalo, D. V., Boyarchuk, A. A., Chechetkin, V. M., Kuznetsov, O. A., \& Molteni, D. 1998, MNRAS, 300, 39

Bisikalo, D. V., Boyarchuk, A. A., Kaigorodov, P. V., Kuznetsov, O. A., \& Matsuda, T. 2004a, Astron. Rep., 48, 449

Bisikalo, D. V., Boyarchuk, A. A., Kaigorodov P. V., Kuznetsov, O. A., \& Matsuda, T. 2004b, Astron. Rep., 48, 588

Bisikalo, D. V., Kononov, D. A., Kaigorodov, P. V., Zhilkin, A. G., \& Boyarchuk, A. A. 2008, Astron. Rep., 52, 318

Boneva, D., Kaigorodov, P. V., Bisikalo, D. V., \& Kononov, D. A. 2009, Astron. Rep., 53, 1004

Gaudenzi, S., Giovannelli, F., Lombardi, R., \& Claudi, R. 1990, AcA, 40, 105

Gaudenzi, S., Giovannelli, F., Mandalari, M., Corradini, M., \& Lombardi, R. 2011, A\&A, 525, A147

Giovannelli, F. 1996, in Multifrequency Behaviour of High Energy Cosmic Sources, ed. F. Giovannelli, \& L. Sabau-Graziati, Mem. SAIt, 67, 401

Giovannelli, F., \& Martinez-Pais, I. G. 1991, SSRv, 56, 313

Giovannelli, F., \& Sabau-Graziati, L. 1998, in Ultraviolet Astrophysics Beyond the IUE Final Archive, ed. W. Wamsteker, \& R. Gonzalez Riestra ESA SP, 413, 419

Gnedin, Yu. N., Natsvlishvili, T. M., Shtol', V. G., Valyavin, G. G., \& Shakhovskoi, N. M. 1995, AstL, 21, 118

Hessman, F. V., Robinson, E. L., Nather, R. E., \& Zhang, E.-H. 1984, ApJ, 286, 747

Kononov, D. A., Kaigorodov, P. V., Bisikalo, D. V., Boyarchuk, A. A., \& Agafonov, M. I. 2008, Astron. Rep., 52, 835

Lucy, L. B. 1994, A\&A, 289, 983

Marsh, T. R., \& Horne, K. 1988, MNRAS, 235, 269

Martinez-Pais, I. G., Giovannelli, F., Rossi, C., \& Gaudenzi, S. 1994, A\&A, 291, 455

Martinez-Pais, I. G., Giovannelli, F., Rossi, C., \& Gaudenzi, S. 1996, A\&A, 308, 833

Merighi, R., Mignoli, M., Ciattaglia, C., et al. 1994, BFOSC: Bologna Faint Object Spectrograph and Camera, Bologna Astronomical Observatory, Internal Report 09-1994-05

North, R. C., Marsh, T. R., Moran, C. K. J., et al. 2001, in Astrotomography, Indirect Imaging Methods in Observational Astronomy, ed. H. M. J. Boffin, D. Steeghs, \& J. Cuypers, LNP, 573, 33

North, R. C., Marsh, T. R., Kolb, U., Dhillon, V. S., \& Moran, C. K. J. 2002, MNRAS, 337, 1215

Papadimitriou, C. J., Harlaftis, E. T., Steeghs, D., \& Niarchos, P. G. 2005, in The Astrophysics of Cataclysmic Variables and Related Objects, ed. J.-M. Hameury, \& J.-P. Lasota, ASP Conf. Proc., 330, 395

Papadaki, C., Boffin, H. M. J., \& Steeghs, D. 2008, JAD, 14, 2

Sawada, K., Matsuda, T., \& Hachisu, I. 1986, MNRAS, 219, 75

Sanad, M. R. 2011, NewA, 16, 114

Storey, P. J., \& Hummer, D. G. 1995, MNRAS, 272, 41

Sytov, A. Yu., Kaigorodov, P. V., Bisikalo, D. V., Kuznetsov, O. A., \& Boyarchuk, A. A. 2007, Astron. Rep., 51, 836

Zhilkin, A. G., \& Bisikalo, D. V. 2011, in 5th International Conference of Numerical Modeling of Space Plasma Flows, ASPC, 444, 91 